\title{
Mindfulness and Acceptance Approaches in Sport Performance
}

\author{
Marjorie Bernier, Emilie Thienot, Romain Codron, \\ and Jean F. Fournier \\ INSEP, French Institute of Sport, Paris
}

\begin{abstract}
The two studies included herein discuss mindfulness and acceptance in sport performance. Based on exploratory interviews with elite swimmers, Study 1 showed that optimal performance, or "flow," states reveal similar characteristics to mindfulness and acceptance states. In flow experiences, the elite swimmers described that they had been particularly mindful of their bodily sensations and accepted them. In Study 2, mindfulness and acceptance were integrated into a psychological skills training program for seven young elite golfers. The program, based on mindfulness and acceptance, contributed to performance enhancement in competition. Participants improved the efficacy of their routines by seeking more relevant internal and external information. The results of both studies corroborated those of previous studies dealing with mindfulness and acceptance in sport. Together, these studies enhance the applicability and efficacy of these approaches with athletic clientele.
\end{abstract}

Mindfulness is defined as the nonjudgmental focus of one's attention on the experience that occurs in the present moment (Kabat-Zinn, 1994). Cottraux (2007) has defined mindfulness as "a mental state resulting from voluntarily focusing one's attention on one's present experience in its sensorial, mental, cognitive and emotional aspects, in a non-judgmental way." Brown and Ryan (2003) have shown that mindfulness is a distinct form of awareness and attention, which could be considered a predisposition for well-being enhancement. Mindfulness has its roots in Eastern meditational practice, and this practice helps to direct attention to the present experience on a moment-to-moment basis (Marlatt \& Kristeller, 1999). Meditation exercises encourage individuals to engage in nonjudging awareness of their internal experience occurring at each moment, such as bodily sensations, cognitions, and emotions and to environmental stimuli, such as sights and sounds (Baer, 2003; Kabat-Zinn, 1994).

Until recently, mindfulness has been a relatively unfamiliar concept in Western culture. Western researchers who have introduced mindfulness practice in mental health treatment programs have taught these skills independently of the religious and cultural traditions of their origin (Kabat-Zinn, 1982; Linehan, 1993). Researchers

The authors are with INSEP, French Institute of Sport, Research Department in Paris, France. 

have developed several clinical interventions based on mindfulness training (KabatZinn, 1982; Teasdale et al., 2000). The targets for its application are mainly, but not only, depressive relapses, anxiety, chronic pain, and posttraumatic stress disorder. These applications belong to the third wave of cognitive-behavioral therapy.

As part of this wave, Hayes and Wilson (1994) introduced Acceptance and Commitment Therapy (ACT). In contrast with other mindfulness-based therapies, ACT has been developed with its own basic research program in human language and cognition: Relational Frame Theory (Hayes, Strosahl, \& Wilson, 1999). ACT is based on the assumption that human language naturally creates psychological suffering, as language itself becomes entangled with actual events, and therefore the language and/or events themselves are responded to as equivalent. The ACT approach combines mindfulness and acceptance skills to help clients develop a new relationship with difficult thoughts, feelings, and emotions, thereby enhancing their psychological health (Harris, 2006).

Recent studies in sport psychology have established a relationship between mindfulness and sport performance (Gardner \& Moore, 2004, 2006; Kee \& Wang, 2008). They have found that mindfulness is linked to present-moment focus, which is the essence of the psychology of peak performance in sport (Jackson \& Csikszentmihalyi, 1999; Ravizza, 2002). Kee and Wang's (2008) study was based on a cluster analytic approach. The authors suggested that athletes who tend to be more mindful are also more likely to experience the flow state. Moreover, they examined the relationships between mindfulness and mental skills adoption. They showed that the high mindfulness clusters scored significantly higher on mental skills adoption measures compared with the low mindfulness clusters, such as on the Attentional Control, Emotional Control, Goal Setting, and Self-Talk subscales of the Test of Performance Strategies (Thomas, Murphy, \& Hardy, 1999).

By combining specific and relevant components from mindfulness and acceptance-based interventions, Gardner and Moore (2004, 2006, 2007) developed a mindfulness- and acceptance-based intervention program for performance enhancement, called the Mindfulness-Acceptance-Commitment (MAC) approach. This manualized program emphasizes nonjudgmental attention to present realities and the development of the skill of self-regulated attention, which is linked with optimal performance. The authors presented two case studies and reported that training in the form of scheduled self-regulation of present-moment awareness enhanced participants' athletic performance and enjoyment (Gardner \& Moore, 2004). Additional open and randomized controlled trials of the MAC program with collegiate athletes has led to enhanced empirical standing for this protocol (Lutkenhouse, Gardner, \& Moore, 2007; Wolanin, 2005).

This recent research has shown that mindfulness and acceptance could be beneficial for performance enhancement, beyond their application for athletes' well-being (Gardner \& Moore, 2004; Kee \& Wang, 2008; Lutkenhouse et al., 2007; Wolanin, 2005). The two studies detailed in the present article sought to further understand the link between mindfulness and acceptance approaches and sport performance. The first study explored the relationship between flow state and mindfulness and acceptance in competitive experiences of elite swimmers. The second study was designed to test the effectiveness of a psychological skills training program based on mindfulness and acceptance over the course of a competitive season. This program was set up for and implemented with young elite golfers. 


\section{Study 1}

A variety of studies have attempted to understand the characteristics of optimal experiences in sport. Several concepts of positive experiences have been described, such as peak experience (Privette, 1981; Ravizza, 1984), peak performance (Cohn, 1991; Privette, 1981) and flow (Csikszentmihalyi, 1975, 1988, 1990). All of these concepts of optimal experience are usually related to expert performance (Singer, 2002). According to Jackson and Roberts (1992), the state of flow in sport represents the psychological state underlying peak performance, which is characterized by the level of accomplishment. The notion of flow also appears to be central in sport psychology to have a better understanding of optimal experiences in sport (Jackson, 2000).

Csikszentmihalyi (1990) defined the concept of flow as an optimal mental state involving total absorption in the task or activity in which one is engaged. Jackson and Csikszentmihalyi (1999) have presented nine characteristics, each representing a distinctive conceptual dimension of flow experience: (a) Challenge-skill balance: a feeling of balance between the demands of the situation and personal skills; (b) Concentration on task at hand: a feeling of being very focused; (c) Merging of action and awareness: a feeling of automaticity about one's action; (d) Clear goals: a feeling of certainty about what one is going to do; (e) Unambiguous feedback: immediate and clear feedback is received, confirming that everything is going according to plan; (f) Sense of total control: a feeling of control that happens without conscious effort; (g) Time transformation: time can be seen more slowly or quickly, or there may be a lack of awareness of the passing of time; (h) Loss of self-consciousness: the person is not concerned by his or her self-judgment or by the judgment of others, and becomes one with the activity; and (i) Autotelic experience: a feeling of pleasure in doing something for its own sake, with no expectation of future reward or benefit. This conceptual model analytically describes the flow experience by dividing it into its different components. To gain greater insight into the nature of flow in sport, this first study was conducted as an exploratory, qualitative investigation of the flow experience of elite swimmers. It sought to understand the mental states of elite swimmers just before and during optimal swimming experiences.

\section{Method}

Participants. The participants were 10 elite swimmers (four women and six men), who were all members of a national French training center. Seven of them were on the French national team and had competed at the international level. The swimmers ranged in age from 18 to 24 years $(M$ age $=20.23, S D=2.87$ ). The sample, due to its international competitive level, offered a rich data source regarding flow experiences, which justified the use of a qualitative inquiry tool (Jackson, 1992).

Procedure. Each participant was asked an identical sequence of questions that were always posed in the same manner. Semistructured, qualitative interviews were used to collect data on flow experience. The interview guide included two 
sections. To prompt the swimmers to describe their flow experiences, they were first asked to describe an optimal experience in their swimming careers, one they would personally consider their most satisfying. Secondly, they were asked to describe a poor swimming experience, one in which they would have expected a better result and they were very disappointed. Swimmers were encouraged to describe in depth both experiences, and specifically their mental states in terms of their cognitions, emotions, and bodily sensations, before and during the performance. The interviewer ensured that all themes were addressed for each participant. Swimmers' citations, drawn directly from transcripts of each interview, became the units of analysis. The interviews, which lasted from 45 to $60 \mathrm{~min}$, were transcribed verbatim. The analyses involved a two-step procedure:

(a) raw data themes were identified from specific swimmers' meaning units; and (b) these raw data themes were organized into interpretable and meaningful themes by an inductive procedure. A deductive analysis was also used to provide a validity check of the results of the inductive analyses.

\section{Results}

Swimmers' descriptions of an optimal swimming experience paralleled many of Jackson and Csikszentmihalyi's dimensions of flow (1999). Some dimensions occurred more frequently than others. Experiences of the autotelic conceptual dimension (e.g., "I felt really happy, and I just wanted to go!") and total concentration (e.g., "I was focused on what I was going to achieve, on what I was going to do") were present in the interviews of all 10 swimmers. Experiences of challenge-skills balance (e.g., "I was confident about what I did before, and I knew that I could do better in the final") were found in 9 of the 10 interviews. Sense of total control (e.g., "I had the sensation of being in control of what I did, so everything seemed easier! I was the one who was totally in charge of what I did, and nobody could change that!") and unambiguous feedback (e.g., "At this time I knew I swam very well. I had very good bodily sensations and my technique was smooth") were present in 8 of the 10 accounts of optimal experiences. Four conceptual dimensions were less common in the interviews: merging of action and awareness (e.g., "I didn't control what I was doing anymore, as if all my movements had become automatic! Everything had became natural") was found in 4 of the 10 interviews, and the last three, clear goals (e.g., "I knew I was there to do the time required, so I only had to fight against myself"), time transformation (e.g., "Indeed, I wasn't aware of time anymore. Everything went very slowly at the beginning ... and everything went so fast after!"), and loss of self-consciousness (e.g., "Just before my race, I felt that I was one with everything...I felt myself in harmony with the environment") were present in only three optimal experiences. In addition to these nine dimensions of the flow experience, one new characteristic emerged in the swimmers' descriptions: the intensity of their bodily sensations, especially before the start of the race. Six of the swimmers spontaneously mentioned a heightened state of bodily awareness during their race preparation. They recalled strong perceptions of bodily sensations as they waited in the marshalling area and just before the start. At such moments, they were highly aware of their internal states and mentioned a strong heartbeat, a "tingling" sensation in their muscles, heat in their extremities, and a feeling of 
"boiling" inside. They also recalled trying not to avoid these unusual bodily sensations even if they were not pleasant, but just to feel and accept them. Some of the swimmers reported the need to focus on their bodily states before the race to be in harmony with themselves and their bodies. Examples include the following:

Then I arrived in the marshalling area, and I felt so much energy inside me. I felt like I was boiling inside . . . my hands were so warm, my whole body was boiling! I felt all this energy so intensely and I accepted it. (Swimmer 7)

Later on, I understood that I just had to totally experience this sensation. . . . This time I felt the tingling, I felt my heart pounding, and I didn't fight it. I didn't move or try to change anything. I let this feeling come. (Swimmer 6)

I could not control anything, at least not my emotions! I was shivering. . . I kept focused on myself ... I'll let everything go. (Swimmer 3)

\section{Discussion}

The purpose of this study was to explore flow experiences in elite swimmers using a qualitative investigation. Although the results were in line with Jackson and Csikszentmihalyi's (1999) model of the nine conceptual dimensions, a new characteristic emerged, which was an awareness and acceptance of their bodily sensations during the preperformance moments.

This particular mental state is comparable to the mindfulness state, which is the nonjudgmental observation of an ongoing stream of internal and external stimuli as they arise (Baer, 2003). The present findings can be compared with the results recently obtained by Kee and Wang (2008). Their study suggests that athletes with the propensity to be more mindful are also more likely to experience the flow state. They chose to examine the issue at a dispositional level because of the difficulty of directly assessing the psychological state of focusing on the present moment while the athletes are in action; together with the possible disruption of their attention on the task at hand. Five out of nine dimensions of flow dispositions were found to be significantly higher for the athletes with the higher mindfulness tendency. According to Kee and Wang (2008), some athletes who are more likely to pay attention to the "here and now" during their course of daily living have a higher chance of experiencing elements of flow more often. Indeed, the authors suggest that the development of mindfulness tendencies may be an indication of flow disposition, but it would be impossible to infer causality between flow and mindfulness. Our present study also emphasizes the link between mindfulness and flow, except that states during experiences have mostly been considered, rather than dispositional and permanent tendencies (traits). Current research has not indicated whether mindfulness can be considered as a trait, a set of skills, or a state (Giluk, 2009). This issue deserves attention to better understand the link between flow and mindfulness.

Moreover, our results solely took into account bodily sensations. Only two participants commented on a conscious state of cognitions and emotions, but this was not specifically investigated. Considering that this study was exploratory, it remains necessary to further examine the link between flow experiences and mindfulness. Moreover, further studies could test the efficacy of a mindfulness-based training on optimal experiences in swimming. 


\section{Study 2}

In the previous study, it appeared that characteristics of mindfulness and acceptance were linked to optimal performance states. Thus it was relevant to further assess the link between mindfulness and acceptance and sport performance by testing the efficacy of an intervention based on mindfulness and acceptance approaches. Hence, the second study focused on how psychological skills training could be improved by integrating a mindfulness and acceptance component.

The efficacy and effectiveness of psychological skills training on performance achievement has often been studied in sport psychology. Numerous articles have addressed this topic since the early published studies in the seventies (e.g., Martin \& Toogood, 1997; Rushall \& Siedentop, 1972; Vealey, 1988; Weinberg, Seabourne, \& Jackson, 1981). More recently, other mental training programs have been established and evaluated (e.g., Curry \& Maniar, 2003; Thelwell, Greenlees, \& Weston, 2006). In the area of golf, Beauchamp, Halliwell, Fournier, and Koestner (1996) implemented a cognitive-behavioral psychological skills training program, adapted from Boutcher and Rotella (1987). They demonstrated that the program was effective at enhancing putting performance. Traditional psychological skills training programs such as goal setting, imagery, arousal control, self-talk, and precompetitive routines have been postulated to help athletes develop psychological skills. These traditional approaches have attempted to achieve ideal performance states through self-control of internal processes such as confidence, attention, emotions, cognitions, and bodily states. Moore (2003) and Gardner and Moore (2004, 2006, 2007) suggested based on theoretical and empirical findings that these traditional psychological skills training processes present some limitations and insufficient efficacy findings. Based on the new perspectives from research in mainstream psychology, their work has suggested that interventions may better target acceptance rather than the direct control of cognitive and affective experiences.

The present study therefore tests the effectiveness of a new psychological skills training program based on mindfulness and acceptance approaches on performance achievement and on the mental skills of young elite golfers. A second aim of the study was to compare the effects of this new program with those of a more traditional program that only combined the development of traditional psychological skills (goal setting, imagery, concentration, and arousal).

\section{Method}

Participants. The participants included seven elite young golfers (two women and five men) from a state golf training center $(M$ age $=15.67, S D=0.74)$. They had practiced golf for 4-10 years. In the training center, they had a daily golf training session that lasted about 3 hours.

Procedure. The golfers underwent the mindfulness and acceptance program from an educational perspective during a complete season. The program was set up after a season during which fundamental mental skills (goal setting, imagery, concentration, relaxation, and activation) were introduced and developed. It was conducted by a researcher with 5 years of experience in teaching psychological skills training procedures to elite amateur golfers. The mindfulness and acceptance 
program was adapted from the structure and the principles of Mindfulnessbased Cognitive Therapy (MBCT; Segal, Williams, \& Teasdale, 2002). This was supplemented with ACT tools developed by Hayes and Strosahl (2004). The use of examples, analogies, and exercises were implemented to describe and enhance the learning of the principles.

The first part of the program consisted of four introductory training sessions from September through December: (a) introduction to mindfulness, focusing on and awareness of breathing and bodily sensations; (b) mindfulness training, focusing on and awareness of breathing, bodily sensations, and movements; (c) mindfulness and acceptance training, awareness of thoughts and emotions in a nonjudgmental way; and (d) mindfulness and acceptance training to set up a three-step process that includes mindfulness, acceptance, and commitment to one's actions by focusing on a relevant object of attention. Each stage lasted one month and was introduced by the researcher during a specific training session. During these sessions, the researcher presented the principles of mindfulness and acceptance using metaphors and then set up specific exercises. Between sessions, the players were instructed to practice a scheduled series of recorded mindfulness and acceptance exercises twice per week using an MP3 player. In addition, they performed an exercise called "body scan" based on MBCT (Segal, Williams, \& Teasdale, 2002) once a day. The goal of this exercise is to scan one's own sensations and cognitions by means of focusing on breathing for about 2 min.

An applied exercise was then implemented during the competition period (second semester). The goal was to integrate the mindfulness and acceptance skills in the preperformance routines of the golfers. In their routines, the first stage consisted of seeking information. By developing mindfulness skills, their awareness of environmental cues (wind, slopes, or hurdles) and internal states (bodily sensations, cognitions) were improved. This allowed them to scan relevant information coming both from their golf environment and from within themselves. This scan was designed to help them be more lucid and to better choose their target, the trajectory, and the required club. The last stage of the routine consisted of focusing on the target, rather than on the stroke. The choice of their external attentional focus was intended to enable them to swing "automatically," without effortful thinking (Beilock, Carr, Mac Mahon, \& Starkes, 2002; Masters, 2000; Wulf \& Prinz, 2001). Their routines integrating mindfulness and acceptance were developed and repeated during golf training sessions. Other applications of mindfulness and acceptance principles in golf situations were introduced in this second part of the program. Thus, some themes linked to the competition context were discussed with the researcher. These themes dealt with, for example, how to perform with negative thoughts (imagining a "failure," feeling "negative" sensations, etc.) and how to play with a high level of anxiety. The researcher used metaphors adapted to a golfing context taken from ACT metaphors (Hayes \& Strosahl, 2004) to teach the principles of mindfulness and acceptance. Contrary to the traditional psychological skills training paradigm, which teaches athletes how to fight or alter their negative cognitions, sensations, and emotions, the metaphors and exercises used in the present program sought to teach how to play golf while experiencing unpleasant or distracting thoughts. This method therefore targeted playing golf with unwanted thoughts to commit to each shot by focusing on the relevant object. This applied work was implemented during competition simulation, training sessions, and direct competitive situations. 
Measures. Outcomes were first assessed with a single-case research design involving behavioral indicators. Behavioral indicators were defined with each golfer at the beginning of the program. Examples of indicators are provided in Table 1. These indicators were related to behaviors during competitions that were linked to performance outcomes in golf. By means of individual interviews, the researcher and the golfers defined the relevant targeted behaviors, which allowed them to examine the effects of mindfulness and acceptance work on their own performance during competition (see Table 1).

Secondly, a longitudinal design was applied using the Ottawa Mental Skills Assessment Tool-3* (OMSAT-3*; Durand-Bush, Salmela, \& Green-Demers, 2001) and qualitative interviews. The OMSAT-3* questionnaire was used to appraise 12 mental skills: goal setting, self-confidence, commitment, stress reactions, fear control, relaxation, activation, imagery, mental practice, focusing, refocusing, and competition planning. The factorial structure of the French version of the OMSAT-3* was evaluated. The 12-factor first-order measurement model provided acceptable goodness-of-fit indices $\left(\chi^{2}=3199.90 ; N=267 ; d f=1014, p<.05\right.$; CFI $=.83$; TLI

$=.81 ;$ RMSEA $=.059 ;$ RMSEA 90\% CI $=.055-.063)$. The OMSAT- $3 *$ results of the golfers were compared with the OMSAT results of six golfers (one woman and five men) from another golf training center $(M$ age $=15.40, S D=0.49)$. This control group followed a traditional mental skills training program conducted by the same researcher. Both groups completed the OMSAT-3* at the beginning and at the end of the season. Repeated-measures ANOVA were conducted to compare the means of both groups (experimental and control groups) under both conditions (at the beginning and at the end of the season) for each skill measured by the OMSAT-3*. Effect sizes were computed using Cohen's $d$ (1988).

Three qualitative interviews were conducted with each golfer and with the coach before the introduction of the intervention, at the end of the first semester (before the competition period), and again at the end of the season. They were mostly used to appraise the understanding and application, in competition, of mindfulness and acceptance principles. Social validation was drawn from qualitative data provided by interviews of the golfers and of their coach.

\section{Table 1 Behavioral Indicators of the Seven Young Elite Golfers}

\begin{tabular}{ll}
\hline Player & Behavioral Indicators \\
A & $\begin{array}{l}\text { Number of distractions during routine (out of } 72 \text { shots) and number } \\
\text { of shots needed to get into a game } \\
\text { B }\end{array}$ \\
C & $\begin{array}{l}\text { of } 72 \text { shots) } \\
\text { Number of routines that were run through too quickly (out of } 72 \text { shots) }\end{array}$ \\
D & $\begin{array}{l}\text { Number of distractions due to noise, number of thoughts focused on } \\
\text { dangerous zones }\end{array}$ \\
E & $\begin{array}{l}\text { Number of hesitations about choices (choices of club, of trajectory) } \\
\text { F }\end{array}$ \\
G & $\begin{array}{l}\text { Percentage of routines that included checking a technical element } \\
\text { during the practice swing }\end{array}$ \\
& $\begin{array}{l}\text { Percentage of routines that included checking the club position } \\
\text { during the practice swing }\end{array}$ \\
\hline
\end{tabular}




\section{Results}

The golfers in the mindfulness and acceptance intervention group all enhanced their national ranking, while only two golfers in the control group did so. Golfers in the intervention group reached their competition goals, as defined at the beginning of the season. They also acquired a sound understanding of mindfulness and acceptance principles. They generally stated that they had developed skills with reference to attentional awareness; a nonjudgmental, task-relevant attentional focus; and behavioral flexibility. For example, according to Participant B, the mindfulness and acceptance training allowed him to more effectively begin his round. Indeed, he enhanced his performance on the first holes. He indicated that he learned to be more aware of his activation level and thus to react faster to variations in activation by playing with it. The coach emphasized that four golfers (Participants A, $\mathrm{B}, \mathrm{C}$, and E) took advantage of their mindfulness and acceptance skills, becoming more aware and lucid in their approach to golf. Regarding social validation, interview analysis indicated that all six participants perceived that they significantly enhanced their performance in competition. In addition, they all noted that they were satisfied with the delivery and content of the program. The coach confirmed that the intervention was useful and relevant. Behavioral indicators defined at the beginning of the intervention turned out to be irrelevant after three competitions. Golfers made quick progress on the targeted behaviors (e.g., a poor decision in choosing a club). They improved so quickly that there was no need to continue working on these issues, hence no formal analyses could be processed to complete the single-case research design.

Statistical analyses on the questionnaire (OMSAT-3*) showed that the golfers who followed the program had increased their scores related to activation, which has been defined as, "the process by which individuals heighten their physiological and mental states in situations where they need to increase their energy, motivation, or focus" (Durand-Bush et al., 2001, p. 5) more than the golfers in the control group, $F(1,10)=6.63, p<.03, d=1.72$.

\section{Discussion}

The purpose of this study was to test the effectiveness of a nontraditional psychological skills training program based on mindfulness and acceptance approaches on the performance and the mental skills of young elite golfers. Results supported the suggestions made by Gardner and Moore $(2004,2006,2007)$ that mindfulness and acceptance approaches may be particularly effective for performance enhancement. The athletes took advantage of this program by developing these new skills that enabled them to be more lucid and focused in competition. Awareness helped them to perceive and manage their activation state more efficiently. When we compared the effects of the mindfulness and acceptance program to those of a traditional, change-based psychological skills training program, statistical results indicated that the mindfulness and acceptance-based program allowed the players to enhance their activation skill significantly more than those who followed the traditional, change-based program.

According to Gardner and Moore (2004, 2006, 2007), athletes must not be considered as a homogeneous sample either by practitioners or by researchers. 
They suggest that mixing nonclinical, subclinical, and clinical participants can cloud important intervention findings, regardless of the type of intervention. They have also shown that using their mindfulness and acceptance based intervention (MAC), which is somewhat similar to the one used here, is effective with nonclinical athletes, and that the addition of certain components is necessary for use with subclinical clients. In the mindfulness and acceptance approaches, individualizing seems to play an important role. Other investigators have emphasized individualizing sport psychology programs to increase their effectiveness (Martin \& Toogood, 1997). While this aspect has not been thoroughly examined in the current study, the mindfulness and acceptance-based program may be enhanced by paying closer attention to personal needs and characteristics.

Difficulties in measuring targeted behavioral indicators arose during the course of this study. Single-case research design methods could be based on more relevant indicators of the performance in question than those selected for the current study. Rather than exclusively examining performance outcomes, it is important to take into account performance subcomponents (Thelwell, Greenlees, \& Weston, 2006). Thus, in golf, technical and tactical subcomponents should be considered. While there is some evidence that mindfulness and acceptance approaches have positive effects on the technical and tactical subcomponents of athletic performance, future research is needed to clarify these effects.

\section{General Discussion and Conclusion}

The goal of these two studies was to examine the link between mindfulness and acceptance approaches and sport performance. Study 1 emphasized the link between flow experience and mindfulness and acceptance states. Some elements of the optimal performance state are comparable to those of the mindfulness and acceptance state. In flow experiences, elite swimmers described that they had been particularly mindful of their bodily sensations and tended to accept them. This characteristic, which seems to reflect a mindfulness and acceptance state, is not clearly described in the nine conceptual dimensions defined by Jackson and Csikszentmihalyi (1999). Future research should further explore the conceptual differences and similarities between flow and mindfulness. In Study 2, mindfulness and acceptance were combined in a psychological skills training program for golfers. Training based on mindfulness and acceptance proved to be effective for enhancing performance during competition for young golfers. The program enabled them to anticipate and adapt their game in a clear-sighted fashion. They improved the efficiency of their routines by seeking more relevant information. Results of the two studies corroborated those of previous studies dealing with mindfulness and acceptance in sport (Gardner \& Moore, 2004, 2006, 2007; Kee \& Wang, 2008). Moreover, this recent research theme in sport psychology shows that mindfulness and acceptance have effects other than simply therapeutic ones, as these skills can be also used for athletic performance enhancement. These approaches, belonging to the third wave of cognitive-behavioral psychology, call into question the singular use of traditional second-wave cognitive-behavioral sport psychology practices that focus of changing or modifying cognitive and affective states.

Sport psychology and performance enhancement interventions have for the most part been based on the second wave of cognitive-behavioral approaches, in 
which cognitions play a central role (Hayes, Strosahl, \& Wilson, 1999). The principle of these traditional methods in sport psychology (as in information processing models, generally) is to detect irrational or ineffective thoughts and to correct them. For example, until now, the awareness skill has been developed as a basis for psychological interventions for performance enhancement. Awareness consists of identifying one's emotional states, arousal level, cognitions, and attentional focus to adjust them. This concept is in one way close to the mindfulness concept, in that it considers both the necessity for an athlete to be lucid and aware of his or her internal states and external cues to achieve greater performance levels. However, mindfulness and acceptance approaches differ from awareness (at least as awareness has been presented in sport psychology; see Ravizza, 2001 for a discussion on awareness) in that mindfulness and acceptance approaches do not seek to control or to change cognitive content. On the contrary, the goal of this third wave approach is to teach athletes to accept their cognitions, emotions, and sensations and to commit themselves to action, rather than fighting against negative thoughts and unpleasant emotions. The difference between awareness, defined by Ravizza as "the recognition of the need to do something to gain control" (2001,p. 171) and mindfulness and acceptance approaches, whose aim is quite the opposite of controlling one's internal state, clearly illustrates the evolution of the principles from the second to the third wave of cognitive-behavioral approaches. This has recently led a handful of researchers and practitioners to reconsider current trends in sport psychology. While the traditional methods may still be useful, they may need to be adapted or adjusted by taking into consideration the new principles of the third wave of cognitive behavioral approaches in mainstream psychology.

More research is needed to gain a better understanding of these approaches and to further confirm their applicability and their efficacy in sport psychology. New investigations should continue to examine mindfulness and acceptance approaches in a variety of sports, as mindfulness and acceptance may require different applications according to the sport in which they are studied and applied. The studies presented here have observed different manifestations and effects of the mindfulness and acceptance process in golf and in swimming. In swimming, mindfulness seemed to help athletes become aware of bodily sensations perceived before the start of the race. In golf, mindfulness and acceptance interventions affected tactical subcomponents of performance. Indeed, mindfulness and acceptance appeared to help the golfers make correct tactical decisions by scanning relevant information coming from internal states (arousal level, emotions) and from the environment (target, potential trajectory of the ball). However, mindfulness and acceptance may present different advantages for different sports, depending on the main subcomponents of performance. In different sports, the optimal performance state displays specific characteristics (i.e., physical, technical, tactical). Thus, it is important to study how mindfulness and acceptance could specifically contribute to the attainment of optimal performance states in various sport contexts.

Interventions must continue to be studied and evolve with emerging data. To date, only two programs have been presented in the literature: the manualized Mindfulness-Acceptance-Commitment (MAC) intervention (Gardner \& Moore, 2004, 2006, 2007), which has garnered initial empirical standing, and the similar program presented in Study 2 of this article. Improvement of the present program implemented with young golfers may include simplification, more sequences, 
and perhaps a shorter duration. New studies should be conducted to enhance our understanding of mindfulness and acceptance in sport, to further confirm the effectiveness of interventions based on mindfulness and acceptance, and to specify how these interventions can be implemented in training athletes at various levels of expertise and across sports.

\section{Acknowledgments}

The authors wish to thank the elite swimmers and golfers who participated in these studies. Study 2 was undertaken with a research grant from the French Federation of Golf and the French Sport Ministry. Thanks to John Salmela and Laura Killian for their comments on the manuscript.

\section{References}

Baer, R.A. (2003). Mindfulness training as a clinical intervention: A conceptual and empirical review. Clinical Psychology: Science and Practice, 10, 125-143.

Beauchamp, P.H., Halliwell, W.R., Fournier, J.F., \& Koestner, R. (1996). Effects of cognitivebehavioral psychological skills training on the motivation, preparation, and putting performance of novice golfers. The Sport Psychologist, 10, 157-170.

Beilock, S.L., Carr, T.H., Mac Mahon, C., \& Starkes, J.L. (2002). When paying attention becomes counterproductive: Impact of divided versus skill-focused attention on novice and experienced performance of sensorimotor skills. Journal of Experimental Psychology. Applied, 8, 6-16.

Boutcher, S.H., \& Rotella, R.J. (1987). A psychological skills educational program for closed-skill performance enhancement. The Sport Psychologist, 1, 127-137.

Brown, K.W., \& Ryan, R.M. (2003). The benefits of being present: Mindfulness and its role in psychological well-being. Journal of Personality and Social Psychology, 84, 822-848.

Cohen, J. (1988). Statistical power analysis for the behavioral sciences (2nd ed.). Hillsdale, NJ: Lawrence Erlbaum Associates.

Cohn, P.J. (1991). An exploratory study on peak performance in golf. The Sport Psychologist, 5, 1-14.

Cottraux, J. (2007). Thérapie cognitive et emotions: La troisième vague [Cognitive therapy and emotions: The third wave]. Paris: Elsevier Masson.

Csikszentmihalyi, M. (1975). Beyond boredom and anxiety the experience of play in work and games. San Francisco: Jossey-Bass Publishers.

Csikszentmihalyi, M. (1988). Optimal experience: Psychological studies of flow in consciousness. Cambridge: Cambridge University Press.

Csikszentmihalyi, M. (1990). Flow: The psychology of optimal experience. New York: Harper \& Row.

Curry, L.A., \& Maniar, S.D. (2003). Academic course combining psychological skills training and life skills education for university students and student-athletes. Journal of Applied Sport Psychology, 15, 270-277.

Durand-Bush, N., Salmela, J.H., \& Green-Demers, I. (2001). The Ottawa Mental Skills Assessment Tool (OMSAT-3*). The Sport Psychologist, 15, 119.

Gardner, F.L., \& Moore, Z.E. (2004). A Mindfulness-Acceptance-Commitment (MAC) based approach to performance enhancement: Theoretical considerations. Behavior Therapy, 35, 707-723.

Gardner, F.L., \& Moore, Z.E. (2006). Clinical sport psychology. Champaign, IL: Human Kinetics.

Gardner, F.L., \& Moore, Z.E. (2007). The psychology of enhancing human performance: The Mindfulness-Acceptance-Commitment (MAC) approach. New York: Springer. 
Giluk, T.L. (2009). Mindfulness, Big Five personality, and affect: A meta-analysis. Personality and Individual Differences, 47, 805-811.

Harris, R. (2006). Embracing your demons: An overview of Acceptance and Commitment Therapy. Psychotherapy in Australia, 12, 2-8.

Hayes, S.C., \& Strosahl, K.D. (2004). A practical guide to acceptance and commitment therapy. New York: Springer.

Hayes, S.C., Strosahl, K.D., \& Wilson, K.G. (1999). Acceptance and Commitment Therapy: An experiential approach to behavior change. New York: Guilford Press.

Hayes, S.C., \& Wilson, K.G. (1994). Acceptance and Commitment Therapy: Altering the verbal support for experiential avoidance. The Behavior Analyst, 17, 289-303.

Jackson, S.A. (1992). Athletes in flow: A qualitative investigation of flow sates in elite figure skaters. Journal of Applied Sport Psychology, 4, 161-180.

Jackson, S.A. (2000). Joy, fun, and flow state in sport. In L.H. Yuri (Ed.), Emotions in sport (pp. 135-155). Champaign: Human Kinetics.

Jackson, S.A., \& Roberts, G. (1992). Positive performance states of athletes: Toward a conceptual understanding of peak performance. The Sport Psychologist, 6, 156-171.

Jackson, S.A., \& Csikszentmihalyi, M. (1999). Flow in sports: The key to optimal experience and performances. Champaign, IL: Human Kinetics.

Kabat-Zinn, J. (1982). An outpatient program in behavioural medicine for chronic pain patients based on the practice of mindfulness meditation: Theoretical considerations and preliminary results. General Hospital Psychiatry, 4, 33-42.

Kabat-Zinn, J. (1994). Wherever you go, there are you: Mindfulness meditation in everyday life. New York: Hyperion.

Kee, Y.H., \& Wang, C.K.J. (2008). Relationships between mindfulness, flow dispositions and mental skills adoption: A cluster analytic approach. Psychology of Sport and Exercise, 9, 393-411.

Linehan, M.M. (1993). Skills training manual for treating borderline personality disorder. New York: Guilford Press.

Lutkenhouse, J., Gardner, F.L., \& Moore, Z.E. (2007). A randomized controlled trial comparing the performance enhancement effects of Mindfulness-Acceptance-Commitment (MAC) performance enhancement and psychological skills training procedures. Manuscript in preparation.

Marlatt, G.A., \& Kristeller, J.L. (1999). Mindfulness and meditation. In W.R. Miller (Ed.), Integrating spirituality into treatment (pp. 67-84). Washington, DC: American Psychological Association.

Martin, G.L., \& Toogood, A. (1997). Cognitive and behavioral components of a seasonal psychological skills training program for competitive figure skaters. Cognitive and Behavioral Practice, 4, 383-404.

Masters, R.S.W. (2000). Theoretical aspects of implicit learning in sport. International Journal of Sport Psychology, 31, 530-541.

Moore, Z.E. (2003). Toward the development of an evidence-based practice of sport psychology: A structured qualitative study of performance enhancement interventions. Dissertation Abstracts International, 64 (10-B), 5227.

Privette, G. (1981). The phenomenology of peak performance in sports. International Journal of Sport Psychology, 12, 51-60.

Ravizza, K. (1984). Qualities of the peak experience in sports. In J. Silva \& R. Weinberg (Eds.), Psychological foundations for sport. Champaign, IL: Human Kinetics.

Ravizza, K. (2002). A philosophical construct: A framework for performance enhancement. International Journal of Sport Psychology, 33, 4-18.

Ravizza, K. (2001). Increasing awareness for sport performance. In J.M. Williams (Ed.), Applied sport psychology: Personal growth to peak performance (pp. 171-181). California: Mayfield Publishing Company.

Rushall, B.S., \& Siedentop, D. (1972). The development and control of behavior in sport and physical education. Philadelphia, PA: Lea \& Febiger. 
Segal, Z.V., Williams, J.M.G., \& Teasdale, J.D. (2002). Mindfulness-based cognitive therapy for depression: A new approach to preventing relapse. New York: Guilford Press.

Singer, R.N. (2002). Preperformance state, routines, and automaticity: What does it take to realize expertise in self-paced events? Journal of Sport \& Exercise Psychology, 24, 359-375.

Teasdale, J.D., Williams, J.M., Soulsby, J.M., Segal, Z.V., Ridgeway, V.A., \& Lau, M.A. (2000). Prevention of relapse/recurrence in major depression by mindfulness-based cognitive therapy. Journal of Consulting and Clinical Psychology, 68, 615-623.

Thelwell, R.C., Greenlees, I.A., \& Weston, N.J.V. (2006). Using psychological skills training to develop soccer performance. Journal of Applied Sport Psychology, 18, 254-270.

Thomas, P.R., Murphy, S.M., \& Hardy, L. (1999). Test of Performance strategies: Development and preliminary validation of a comprehensive measure of athletes' psychological skills. Journal of Sports Sciences, 17, 697-711.

Vealey, R.S. (1988). Future directions in psychological skills training. The Sport Psychologist, 2, 318-336.

Weinberg, R.S., Seabourne, T.G., \& Jackson, A. (1981). Effects of visuo-motor behavior rehearsal, relaxation, and imagery on karate performance. Journal of Sport Psychology, 3, 228-238.

Wolanin, A.T. (2005). Mindfulness-acceptance-commitment (MAC) based performance enhancement for Division I collegiate athletes: A preliminary investigation. Dissertation Abstracts International, 65 (7-B), 3735.

Wulf, G., \& Prinz, W. (2001). Directing attention to movement effects enhances learning: A review. Psychonomic Bulletin \& Review, 8, 648-660. 